\title{
Cas de VIH nouvellement diagnostiqués chez les personnes de 50 ans et plus, comparativement aux personnes de moins de 50 ans : 2008 à 2017
}

\author{
N Haddad ${ }^{1 \star}$, A Robert ${ }^{1}$, N Popovic ${ }^{1}$, O Varsaneux ${ }^{1}$, M Edmunds ${ }^{1}$, L Jonah 1 , W Siu ${ }^{1}$, A Weeks ${ }^{1}$, \\ C Archibald ${ }^{1}$
}

\section{Résumé}

Contexte : La population canadienne vieillit et près de $40 \%$ des Canadiens sont âgés de 50 ans et plus. Avec le vieillissement de la population, des problèmes uniques liés à la santé commencent à se manifester, notamment le nombre croissant d'infections transmissibles sexuellement et par le sang. Comprendre l'épidémiologie du VIH chez les personnes plus âgées est important en vue d'orienter les programmes de prévention des maladies et de lutte contre celles-ci.

Objectif : Évaluer les tendances en matière de cas de VIH nouvellement diagnostiqués au Canada chez les personnes de 50 ans et plus ( $\geq 50$ ans) et celles de moins de 50 ans ( $<50$ ans), puis comparer leurs caractéristiques démographiques de base et leurs catégories d'exposition pour la période de 2008 à 2017.

Méthodes : La surveillance du VIH à l'échelle nationale est menée par l'Agence de la santé publique du Canada par l'entremise de la communication (à titre volontaire) de données par les autorités de santé publique provinciales et territoriales. Des analyses descriptives ont été menées sur les cas de VIH signalés

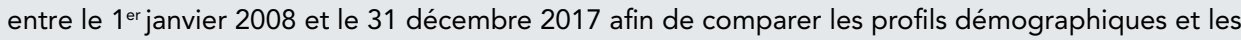
catégories d'exposition pour les deux groupes d'âge.

Résultats : Entre 2008 et 2017, la proportion des cas de VIH nouvellement diagnostiqués chez les 50 ans et plus est passée de 15,1 \% à 22,8\%. Les taux de diagnostic du VIH chez les hommes et les femmes plus âgés ont augmenté avec le temps, et une augmentation relativement plus élevée a été constatée chez les femmes. Une proportion plus élevée de cas de VIH nouvellement diagnostiqués étaient des hommes dans le groupe des plus âgés $(81,2 \%)$ par rapport au groupe plus jeune $(74,6 \%)$. La catégorie d'exposition la plus courante regroupait les groupes jeunes et plus âgés d'hommes gais, bisexuels et autres hommes ayant eu des relations sexuelles avec des hommes (gbHARSAH). Viennent ensuite les catégories de contacts hétérosexuels et de consommation de drogues injectables; cependant, les proportions relatives variaient selon l'âge, puisque la catégorie de gbHARSAH était plus élevée dans le groupe des moins de 50 ans.

Conclusion : Au Canada, plus de $20 \%$ de tous les cas de VIH nouvellement diagnostiqués concernent maintenant des personnes âgées de 50 ans et plus. Les initiatives de dépistage et de prévention du VIH visent depuis toujours les populations plus jeunes et pourraient ne pas avoir la même incidence sur les populations plus âgées. Ces données peuvent être utilisées pour orienter les futures mesures de santé publique qui seront conçues pour lutter contre le VIH chez les populations plus âgées.
Cette oeuvre est mise à la disposition selon les termes de la licence internationale Creative Commons Attribution 4.0

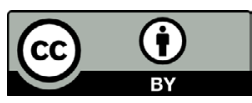

Affiliation

${ }^{1}$ Le Centre de la lutte contre les maladies transmissibles et les infections, l'Agence de la Santé publique Canada,

Ottawa, ON

\section{*Correspondance:}

phac.hass.aspc@canada.ca
Citation proposée : Haddad N, Robert A, Popovic N, Varsaneux O, Edmunds M, Jonah L, Siu W, Weeks A, Archibald C. Cas de VIH nouvellement diagnostiqués chez les personnes de 50 ans et plus, comparativement aux personnes de moins de 50 ans : 2008 à 2017. Relevé des maladies transmissibles au Canada 2019;45(11):311-7. https://doi.org/10.14745/ccdr.v45i11a02f

Mots-clés : VIH, surveillance, gais, bisexuels, hommes ayant des relations sexuelles avec des hommes, utilisateurs de drogues injectables, 50 ans et plus 


\section{Introduction}

À l'échelle mondiale, on estimait à 36,9 millions le nombre de personnes vivant avec le VIH en 2017 (1), et on évalue que plus de $10 \%$ de la population adulte vivant avec le VIH dans les pays à revenu faible ou intermédiaire est âgée de 50 ans ou plus ( $\geq 50$ ans) (2). Dans les pays développés, ces personnes âgées de $\geq 50$ ans représentent environ $30 \%$ des adultes atteints du VIH (3). En raison des progrès constants réalisés dans le traitement du $\mathrm{VIH}$, le nombre et la proportion de personnes vivant avec le $\mathrm{VIH}$ à un âge avancé vont probablement continuer d'augmenter (4); on prévoit que le nombre de personnes âgées de $\geq 50$ ans atteintes du $\mathrm{VIH}$ et ayant un revenu faible ou intermédiaire augmentera de $47 \%$, pour atteindre 6,9 millions d'ici 2020 (5).

La proportion de la population canadienne âgée de $\geq 50$ ans a augmenté de $12,7 \%$ au cours des cinq dernières années et atteint maintenant près de $40 \%$ (6). À mesure que le nombre d'aînés canadiens augmentera et qu'ils vivront plus longtemps, ils seront confrontés à plusieurs enjeux liés au maintien et à la promotion de la santé. Les Canadiens plus âgés qui sont atteints du VIH vivent également avec la possibilité de faire face à : un vieillissement accéléré, des effets secondaires causés par I'utilisation prolongée d'antirétroviraux (7) et des taux plus élevés de comorbidités non liées au sida, notamment le diabète, le cancer et les maladies cardiovasculaires $(4,8)$. Ainsi, l'épidémie de $\mathrm{VIH}$ pose de graves problèmes aux personnes plus âgées en raison des problèmes physiques, mentaux et psychologiques qui accompagnent inévitablement le vieillissement, aussi bien que I'infection au VIH $(9,10)$. Avec le vieillissement des populations nationales et mondiales, il est important de comprendre l'épidémiologie du VIH chez les personnes plus âgées en vue d'orienter les programmes de sensibilisation et de prévention.

L'objectif de la présente analyse consiste à évaluer les tendances des cas de VIH nouvellement diagnostiqués au Canada pour les populations âgées de $\geq 50$ ans et celles de moins de 50 ans ( $<50$ ans), puis de comparer les caractéristiques démographiques de base ainsi que les catégories d'exposition de ces deux populations, pour la période de 2008 à 2017.

\section{Méthodes}

L'étude s'appuie sur des données du Système national de surveillance du VIH/sida, qui est géré par l'Agence de la santé publique du Canada (ASPC) (11). Le Système national de surveillance du VIH/sida est un système passif basé sur des cas, qui collige des données non nominales de personnes nouvellement diagnostiquées séropositives au Canada; ces données sont soumises à l'ASPC par les autorités de santé publique provinciales et territoriales. Les détails relatifs aux méthodes de surveillance ont été décrits précédemment $(11,12)$.
Les données relatives à l'âge, au sexe et aux catégories d'exposition ont été analysées pour les cas de VIH signalés entre le $1^{\text {er }}$ janvier 2008 et le 31 décembre 2017. Les cas ont été stratifiés en deux groupes : un composé de personnes de $\geq 50$ ans au moment du diagnostic; et un composé de celles de $<50$ ans au moment du diagnostic. Les catégories d'exposition utilisées dans la surveillance du VIH sont indiquées au tableau 1 et sont utilisées selon un cadre hiérarchique (11) afin de refléter le mode de transmission du VIH le plus probable, même si un cas donné comportait plusieurs catégories d'exposition.

\section{Tableau 1 : Liste des catégories d'exposition et des descriptions utilisées dans la surveillance nationale du VIH (11)}

\begin{tabular}{|c|c|}
\hline Catégorie d'exposition & Type d'exposition signalé \\
\hline $\begin{array}{l}\text { Gai, bisexuel et autres hommes } \\
\text { ayant eu des relations sexuelles } \\
\text { avec des hommes (gbHARSAH) }\end{array}$ & Rapport sexuel homosexuel \\
\hline $\begin{array}{l}\text { Utilisateurs de drogues injectables } \\
\text { (UDI) }\end{array}$ & Usage de drogues par injection \\
\hline gbHARSAH-UDI & $\begin{array}{l}\text { Rapport sexuel homosexuel et } \\
\text { usage de drogues injectables }\end{array}$ \\
\hline $\begin{array}{l}\text { Hétérosexuel/endémique }{ }^{a} \\
\text { (Hét-endémique) }\end{array}$ & $\begin{array}{l}\text { Une personne née dans un } \\
\text { pays où le VIH est endémique } \\
\text { qui rapporte des rapports } \\
\text { hétérosexuels }\end{array}$ \\
\hline $\begin{array}{l}\text { Hétérosexuel/risque } \\
\text { (Hét-risque) }\end{array}$ & $\begin{array}{l}\text { Relations sexuelles } \\
\text { hétérosexuelles avec une } \\
\text { personne infectée par le VIH } \\
\text { ou présentant un risque accru } \\
\text { d'infection par le VIH (p. ex. } \\
\text { une personne qui s'injecte des } \\
\text { drogues, un homme qui a des } \\
\text { partenaires sexuels masculins ou } \\
\text { une personne originaire d'un pays } \\
\text { où le VIH est endémique) }\end{array}$ \\
\hline $\begin{array}{l}\text { Hétérosexuel/aucun risque signalé } \\
\text { (ARS-Hét) }\end{array}$ & $\begin{array}{l}\text { Relations sexuelles } \\
\text { hétérosexuelles, lorsque le } \\
\text { contact hétérosexuel est le seul } \\
\text { facteur de risque signalé et que } \\
\text { rien n'est connu en ce qui a trait } \\
\text { aux facteurs liés au VIH en lien } \\
\text { avec le partenaire }\end{array}$ \\
\hline Autres & $\begin{array}{l}\text { Exposition périnatale, exposition } \\
\text { par le travail ou transfusion } \\
\text { de sang ou de facteurs de } \\
\text { coagulation hors du pays, et toute } \\
\text { autre sorte d'exposition }\end{array}$ \\
\hline \multicolumn{2}{|c|}{$\begin{array}{l}\text { a'Agence de la santé publique du Canada définit les pays où le VIH est endémique comme } \\
\text { ceux où la prévalence du VIH chez les adultes (âgés de } 15 \text { à } 49 \text { ans) est d'au moins } 1,0 \% \text { et } \\
\text { qu'au moins un des critères suivants s'applique : } 50 \% \text { ou plus des cas de VIH nouvellement } \\
\text { diagnostiqués sont attribués à la transmission hétérosexuelle; un ratio hommes-femmes de } 2: 1 \\
\text { ou moins, parmi les infections prévalentes; ou un taux de prévalence du VIH supérieur ou égal à } \\
2 \% \text { chez les femmes bénéficiant de soins prénatals }\end{array}$} \\
\hline
\end{tabular}

Le Québec ne soumet pas de données sur la catégorie d'exposition à l'ASPC, de sorte que les cas nouvellement diagnostiqués au Québec ont été exclus des analyses impliquant 
la catégorie d'exposition. Des tests du chi carré séparés ont été menés pour comparer la répartition des catégories d'exposition entre les groupes plus âgés et les plus jeunes, aussi bien chez les hommes que chez les femmes. Tous les taux ont été calculés à partir des dénominateurs de population, lesquels ont été recueillis dans la publication de Statistiques démographiques annuelles produite par Statistique Canada (13).

\section{Résultats}

II y a eu 22534 cas de VIH nouvellement diagnostiqués, entre 2008 et 2017 , et pratiquement tous les cas (99,8\%) comprenaient des données sur l'âge ( $n=22486)$. Dans l'ensemble, $19,7 \%$ de ces habitants ( $n=4438$ ) étaient âgées de $\geq 50$ ans, représentant une augmentation de 39,1\%. Le nombre de nouveaux diagnostics de $\mathrm{VIH}$ chez les $\geq 50$ ans est passé de 15,1\% en 2008 à 22,8 \% en 2017 (figure 1). De même, le taux de diagnostics de $\mathrm{VIH}$ pour cette tranche d'âge est passé de 3,5 pour 100000 habitants en 2008 à 3,9 pour 100000 en 2017. Le taux de diagnostic de VIH pour le groupe plus jeune était plus élevé que pour le groupe des plus âgés, et il a fluctué au cours de cette période, mais a diminué de 9,9 pour 100000 habitants en 2008 à 8,0 pour 100000 habitants en 2017.

\section{Figure 1 : Nombres et taux rapportés (pour} 100000 habitants) concernant les diagnostics de VIH chez les personnes plus âgées ( $\geq 50$ ans), chez les plus jeunes ( $<50$ ans) et chez tous les Canadiens dont l'âge a été déclaré, 2008 à $2017^{a}$

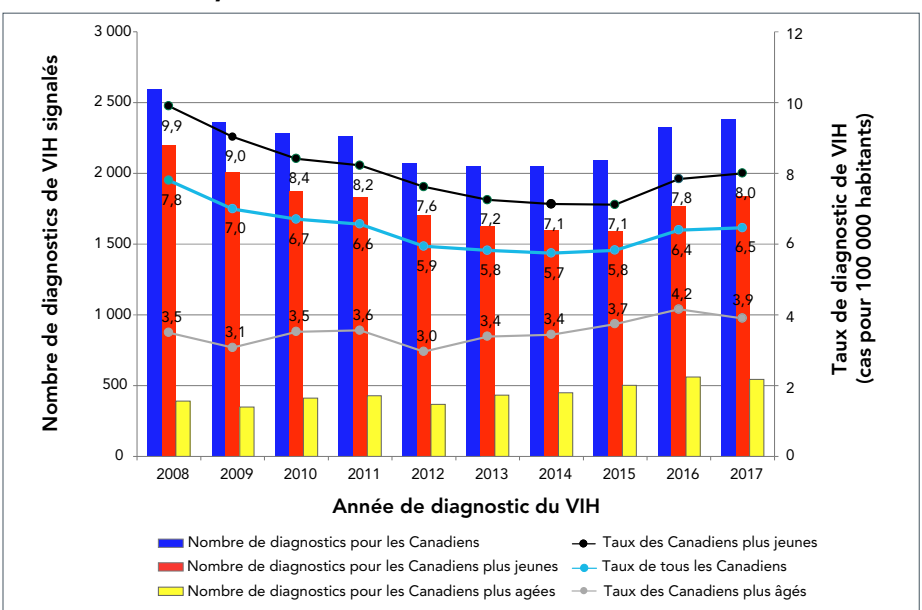

Source des données sur la population : Statistiques démographiques annuelles produites par Statistique Canada (13)

Exclut les cas dont l'âge est inconnu au moment du diagnostic de VIH

\section{Répartition par groupe d'âge et sexe}

De 2008 à 2017, des données relatives à l'âge et au sexe étaient disponibles pour 22413 (99,4\%) des 22534 cas de $\mathrm{VIH}$ nouvellement diagnostiqués. Parmi ceux-ci, 81,2\% étaient des hommes âgés de $\geq 50$ ans, contre $74,6 \%$ qui étaient des hommes âgés de $<50$ ans (données non présentées). Les taux correspondants de dia+/gnostic du VIH étaient plus élevés chez les hommes que chez les femmes, et ce, pour les deux groupes d'âge. Les taux chez les hommes et les femmes âgés de $\geq 50$ ans ont augmenté avec le temps, la hausse relative étant plus élevée chez les femmes (passant de 1,0 à 1,6 pour 100000 habitants chez les femmes, et de 6,6 à 6,5 pour 100000 habitants chez les hommes). Chez les $<50$ ans, les taux tant chez les hommes que chez les femmes ont diminué avec le temps, la baisse étant proportionnellement plus forte chez les femmes (passant de 5,6 à 4,2 pour 100000 habitants chez les femmes, et de 14,1 à 11,7 pour 100000 habitants chez les hommes) (figure 2).

Figure 2 : Taux annuels (pour 100000 habitants) de nouveaux diagnostics de VIH par tranche d'âge et par sexe, Canada, 2008 à $2017^{a, b}$

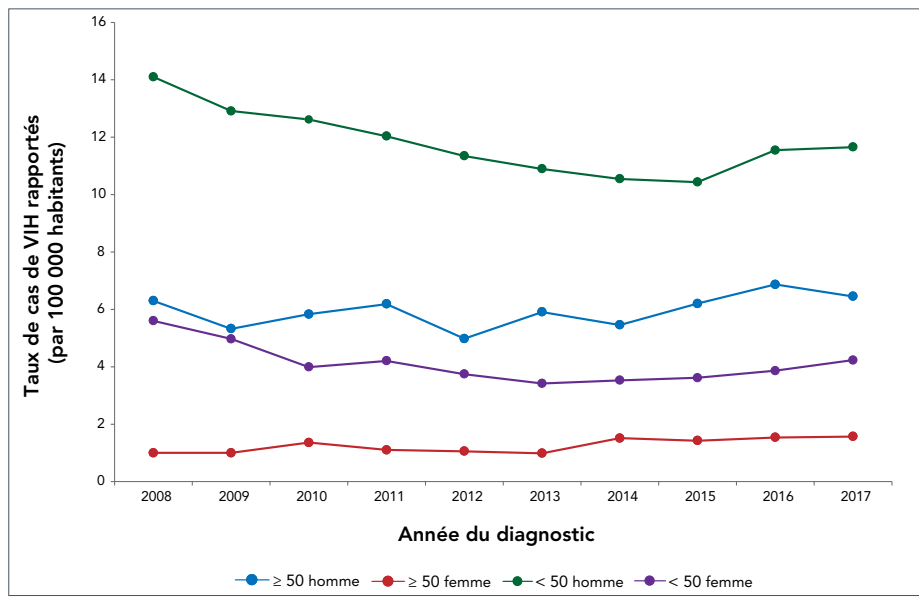

Abréviations : $\geq 50$, âgé de 50 ans et plus; $<50$, âgé de moins de 50 ans

a Source des données sur la population: Statistiques démographiques annuelles produites par Statistique Canada (13)

b Exclut les cas dont l'âge est inconnu au moment du diagnostic de $\mathrm{VIH}$. Exclut les cas où le sexe est transgenre, transsexuel, autre, inconnu ou non rapporté

\section{Répartition des catégories d'exposition}

De 2008 à 2017, il y a eu 14751 nouveaux cas de diagnostics de VIH où l'âge, le sexe et la catégorie d'exposition étaient connus (65\%). Étant donné que la catégorie d'exposition des hommes gais, bisexuels et autres hommes ayant des rapports sexuels avec des hommes (gbHARSAH) ne s'appliquait qu'aux hommes, I'analyse de la variable relative à la catégorie d'exposition a été réalisée séparément, par sexe. Autant chez les hommes plus âgés que chez les plus jeunes, le groupe gbHARSAH constituait la catégorie d'exposition la plus courante, suivie par la catégorie des contacts hétérosexuels, puis la catégorie d'usage de drogues par injection. Par ailleurs, plus d'hommes jeunes $(62,4 \%)$ se sont identifiés à la catégorie gbHARSAH, que d'hommes plus âgés (48,0\%). En revanche, il y avait moins d'hommes qui se sont identifiés à la catégorie des contacts hétérosexuels dans le groupe plus jeune $(18,3 \%)$ comparativement au groupe plus âgé $(32,0 \%)$. La proportion attribuée à la catégorie d'usage de drogues par injection était semblable dans les deux groupes: $14,4 \%$ parmi les hommes plus âgés; et 12,5\% parmi les hommes plus jeunes (tableau 2).

Chez les femmes, la catégorie relative aux contacts hétérosexuels était la plus élevée dans les deux groupes d'âge, mais elle était relativement plus élevée chez les $\geq 50$ ans $(75,1 \%$ contre $63,1 \%)$. Par ailleurs, la proportion de cas dans la 
catégorie d'exposition relative à l'usage de drogues injectables était relativement plus élevée chez les $<50$ ans $(29,0 \%$ contre $16,0 \%$ ). Dans la catégorie d'exposition relative aux contacts hétérosexuels, la proportion attribuée aux personnes en provenance de pays où le $\mathrm{VIH}$ est endémique était semblable pour les deux groupes d'âge $(25,8 \% \geq 50$ ans; $28,0 \%<50$ ans) (tableau 2). La répartition des diagnostics de $\mathrm{VIH}$ pour l'ensemble des catégories d'exposition était considérablement différente entre les populations plus jeunes et celles plus âgées, et ce, aussi bien chez les hommes que chez les femmes (test du chi carré; $p<0,0001)$.

\section{Tableau 2 : Nombre et proportion de cas de VIH nouvellement diagnostiqués par groupe d'âge, sexe et catégorie d'exposition-Canada (à l'exclusion du Québec), 2008 à $2017^{\mathrm{a}-\mathrm{c}}$}

\begin{tabular}{|c|c|c|c|c|c|c|c|c|}
\hline \multirow{3}{*}{$\begin{array}{c}\text { Catégorie } \\
\text { d'exposition }\end{array}$} & \multicolumn{4}{|c|}{ Hommes } & \multicolumn{4}{|c|}{ Femmes } \\
\hline & \multicolumn{2}{|c|}{$\begin{array}{l}\text { Âgé de } \\
\text { moins de } \\
50 \text { ans }\end{array}$} & \multicolumn{2}{|c|}{$\begin{array}{l}50 \text { ans et } \\
\text { plus }\end{array}$} & \multicolumn{2}{|c|}{$\begin{array}{l}\text { Âgé de } \\
\text { moins de } \\
50 \text { ans }\end{array}$} & \multicolumn{2}{|c|}{$\begin{array}{c}50 \text { ans et } \\
\text { plus }\end{array}$} \\
\hline & $\mathrm{n}$ & $\%$ & $\mathrm{n}$ & $\%$ & $\mathrm{n}$ & $\%$ & $n$ & $\%$ \\
\hline gbHARSAH & 5723 & 62,4 & 957 & 48,0 & $\mathrm{~S} / \mathrm{O}$ & 0,0 & $\mathrm{~S} / \mathrm{O}$ & 0,0 \\
\hline $\begin{array}{l}\text { gbHARSAH- } \\
\text { UDI }\end{array}$ & 348 & 3,8 & 47 & 2,4 & $\mathrm{~S} / \mathrm{O}$ & 0,0 & $\mathrm{~S} / \mathrm{O}$ & 0,0 \\
\hline UDI & 1150 & 12,5 & 288 & 14,4 & 898 & 29,0 & 78 & 16,0 \\
\hline Autres $^{\mathrm{a}}$ & 277 & 3,0 & 63 & 3,2 & 244 & 7,9 & 44 & 9,0 \\
\hline $\begin{array}{l}\text { Contact } \\
\text { hétérosexuel }\end{array}$ & 1675 & 18,3 & 639 & 32,0 & 1953 & 63,1 & 367 & 75,1 \\
\hline $\begin{array}{l}\text { Hét- } \\
\text { endémique }\end{array}$ & 522 & 5,7 & 121 & 6,1 & 867 & 28,0 & 126 & 25,8 \\
\hline Hét-risque & 464 & 5,1 & 240 & 12,0 & 620 & 20,0 & 129 & 26,4 \\
\hline ARS-Hét & 689 & 7,5 & 278 & 13,9 & 466 & 15,1 & 112 & 22,9 \\
\hline Sous-total $\left.\right|^{b}$ & 9173 & - & 1994 & - & 3095 & - & 489 & - \\
\hline $\begin{array}{l}\text { Aucun risque } \\
\text { signalé }\end{array}$ & 403 & 3,0 & 152 & 4,2 & 143 & 3,1 & 27 & 3,2 \\
\hline $\begin{array}{l}\text { Catégorie } \\
\text { d'exposition } \\
\text { inconnue } \\
\text { ou non } \\
\text { rapportée } \\
\text { (manquante) }\end{array}$ & 3850 & 28,7 & 1448 & 40,3 & 1322 & 29,0 & 317 & 38,1 \\
\hline Total & 13426 & - & 3594 & - & 4560 & - & 833 & \\
\hline
\end{tabular}

Abréviations : ARS-hét, hétérosexuel/aucun risque signalé; gbHARSAH, gais, bisexuels et autres hommes ayant des relations sexuelles avec des hommes; Hét-endémique, hétérosexuel/ endémique; ; Hét-risque, hétérosexuel/risque; S.O., ou (-), sans objet; UDI, utilisateurs de drogues injectables

a "Autres » comprend la coagulation, le sang, l'exposition par le travail, à l'extérieur du pays, la périnatalité, les expositions au sang / coagulation et autres

bemprend uniquement les cas où l'âge au moment du diagnostic de $\mathrm{VIH}$, le sexe ainsi que la catégorie d'exposition sont connus et rapportés

' Le test du chi carré était concluant $(p<0,0001)$ au moment de comparer les groupes d'âge chez les hommes plus âgés et ceux plus jeunes, ainsi que chez les femmes plus âgées et celles plus jeunes

De 2008 à 2017, la répartition des catégories d 'exposition par groupe d'âge n'a pas beaucoup changé pour les hommes et les femmes (données non présentées). Par exemple, chez les hommes, le groupe gbHARSAH a continué de représenter la plus importante catégorie d'exposition au fil du temps, tandis que chez les femmes, ce sont les contacts hétérosexuels qui ont continué de représenter la plus importante catégorie d'exposition.

\section{Discussion}

Au Canada, plus de $20 \%$ de tous les cas de VIH nouvellement diagnostiqués concernent maintenant des personnes âgées de 50 ans et plus. Le nombre de nouveaux diagnostics de $\mathrm{VIH}$ chez les personnes plus âgées a augmenté de 39,1\% entre 2008 et 2017; de même, le taux de nouveaux diagnostics de $\mathrm{VIH}$ au cours de cette période a également augmenté. Cette tendance contraste avec la catégorie des plus jeunes, qui a connu une diminution globale de la proportion et du taux de nouveaux diagnostics de VIH au cours de la même période; ces changements au fil du temps ont été observés à la fois chez les hommes et chez les femmes, bien que les changements relatifs aient été observés de façon plus importante chez les femmes. La plus grande proportion de nouveaux diagnostics a été constatée dans la catégorie d'exposition gbHARSAH, représentant près de la moitié des hommes plus âgés et les deux tiers des hommes plus jeunes.

La tendance à la hausse des diagnostics de VIH chez les personnes plus âgées a également été observée dans d'autres pays. L'incidence de $20 \%$ se situe entre les estimations mondiales de I'ONUSIDA de $10 \%$ (pour les pays à revenu faible et intermédiaire) (2) et $30 \%$ (pour les pays développés) (3). Au Royaume-Uni, le nombre de nouveaux cas d'infections au $\mathrm{VIH}$ a diminué de façon générale; par contre, le nombre et la proportion de nouveaux diagnostics chez les personnes de $\geq$ 50 ans ont continué d'augmenter (14). À titre comparatif, une analyse récente des données du Système de surveillance européenne a comparé les personnes plus âgées ( $\geq 50$ ans) aux personnes plus jeunes (15 à 49 ans) en 2014 et 2015. Le taux de notification moyen des nouveaux diagnostics chez les personnes plus âgées a augmenté, principalement dans les pays de l'Union européenne de l'Est et de l'Espace économique européen. L'analyse a également révélé que les personnes âgées de $\geq 50$ ans sont plus susceptibles de contracter le VIH par contact hétérosexuel et de présenter des signes de la maladie tardivement (15). Aux États-Unis, en revanche, les diagnostics de $\mathrm{VIH}$ chez les personnes de $\geq 50$ ans ont diminué de $7 \%$, entre 2011 et 2015 (16). Cette tendance a également été observée dans la ville de New York, où le nombre de nouveaux diagnostics de VIH, de 2001 à 2017, a diminué de manière significative pour l'ensemble de la population et pour les personnes âgées de $\geq 50$ ans (17).

Une augmentation des tests de dépistage du VIH pourrait expliquer en partie l'augmentation du nombre de diagnostics de VIH attribués aux Canadiens plus âgés. La Colombie-Britannique a connu une hausse générale de la fréquence des tests de dépistage du VIH depuis 2011, et la fréquence des tests dépistage des $\geq 50$ ans a augmenté, de 2011 à 2016 (18). Une forte proportion de nouveaux diagnostics ont été rapportés dans la catégorie d'exposition gbHARSAH (près de la moitié chez les hommes plus âgés et les deux tiers chez les hommes plus jeunes), qui demeure le principal moteur de l'épidémie de $\mathrm{VIH}$ au Canada (12). En général, la population gbHARSAH continue 
d'être touchée de façon disproportionnée par le VIH. En Ontario, le pourcentage de tests dans la population gbHARSAH est passé de 17,7 \% en 2007 à $28,6 \%$ en 2016 (19). Les tests peuvent également inclure les hommes gbHARSAH séronégatifs pour le VIH, groupe qui présente un risque plus élevé, car le test constitue la première étape pour envisager des approches préventives telles que la prophylaxie préexposition (20).

Outre ces initiatives de dépistage, plusieurs autres raisons pourraient expliquer l'augmentation des proportions et des taux chez les personnes âgées de $\geq 50$ ans, notamment le faible usage du préservatif chez les personnes plus âgées (21-23); le fait de ne pas mettre en priorité les efforts de prévention du $\mathrm{VIH}$ chez les adultes plus âgés (24); et une tendance à obtenir un diagnostic de façon relativement tardive au sein de cette population (23), permettant ainsi la transmission du virus. Aux États-Unis, les personnes plus âgées ( $\geq 50$ ans) sont davantage susceptibles d'être atteintes du VIH à un stade avancé de I'infection au moment du diagnostic, et donc de commencer le traitement de manière tardive (16). Les personnes plus âgées sont susceptibles de souffrir de troubles médicaux liés au VIH plus tard au cours de la maladie, comparativement à leurs homologues plus jeunes (25). Au Canada, le nombre de diagnostics de femmes âgées de $\geq 50$ ans a augmenté depuis 2008 , et les contacts hétérosexuels constituaient la catégorie d'exposition la plus fréquemment signalée pour ce groupe d'âge (ainsi que pour les femmes plus jeunes).

Cette augmentation du nombre de cas de VIH diagnostiqués chez les femmes plus âgées pourrait être due en partie au fait que ces femmes sont moins préoccupées par les risques de grossesse et s'adonnent possiblement davantage à des rapports sexuels non protégés (22,26-28). La hausse observée des diagnostics de $\mathrm{VIH}$ rapportés chaque année pourrait également être attribuée en partie à des diagnostics antérieurs d'infection par le VIH, pour lesquels une personne aurait pu être testée et ce nouveau cas aurait été rapporté plusieurs fois, et ce, en raison de la migration interprovinciale ou encore de la migration au Canada après avoir reçu un diagnostic de $\mathrm{VIH}$ à l'extérieur du Canada. Cependant, pour le moment, il n'est pas possible d'évaluer l'incidence d'un diagnostic reçu antérieurement par groupe d'âge, car cette information n'est pas disponible. L'augmentation du nombre de cas de VIH nouvellement diagnostiqués chez les adultes de $\geq 50$ ans entraîne des répercussions importantes. Les personnes plus âgées présentent des caractéristiques démographiques et des facteurs de risque différents de ceux des adultes plus jeunes; ainsi, les initiatives de dépistage et de prévention du $\mathrm{VIH}$, qui ont toujours visé une population plus jeune, pourraient ne pas avoir la même efficacité sur les populations plus âgées. Prévoir des interventions pour cibler les caractéristiques distinctives de cette population plus âgée pourrait s'avérer nécessaire. L'Agence de la santé publique du Canada continuera de fournir des mises à jour concernant la situation du $\mathrm{VIH}$ au sein de cette population, car ces données pourraient être utilisées en vue d'orienter les futures mesures de santé publique qui seront conçues pour lutter contre le $\mathrm{VIH}$ chez les personnes plus âgées.

\section{Limites}

Bien que les données de surveillance décrivent les cas diagnostiqués de l'épidémie, les estimations nationales de la prévalence et de l'incidence du VIH fournissent un portrait global de l'épidémie de VIH au Canada et incluent les personnes infectées par le VIH qui ont été diagnostiquées et non diagnostiquées (29). Les données relatives aux nouveaux diagnostics ne concernent que les individus testés et diagnostiqués séropositifs, et ne représentent aucunement tout l'éventail des personnes vivant avec le $\mathrm{VIH}$. De plus, ces données ne fournissent aucun renseignement quant au moment où ces personnes ont été infectées. L'analyse décrite dans la présente étude s'est limitée à des variables présentant des données relativement complètes, notamment l'âge, le sexe et la catégorie d'exposition. Enfin, ces données pourraient inclure un sous-groupe de personnes ayant reçu un diagnostic de $\mathrm{VIH}$ dans une autre province (ou autre pays). D'autres limites relatives au Système national de surveillance du $\mathrm{VIH} /$ sida ont été décrites ailleurs dans le présent document (12).

\section{Conclusion}

Au Canada, plus de $20 \%$ de tous les cas de VIH nouvellement diagnostiqués concernent maintenant des personnes âgées de 50 ans et plus. Les initiatives nationales de dépistage et de prévention du VIH visent depuis toujours les populations plus jeunes et pourraient ne pas avoir la même incidence sur les populations plus âgées. Ces données peuvent être utilisées pour orienter les futures mesures de santé publique qui seront conçues pour lutter contre le $\mathrm{VIH}$ chez les populations plus âgées.

\section{Déclaration des auteurs}

N. H. - Acquisition de données, analyse et interprétation de données, réécriture de l'article original, notamment la conceptualisation, rédaction, version définitive, révision, édition, validation, visualisation

A. R. - Acquisition et analyse de données, validation, rédaction et révision de la version définitive

N. P. - Révision, édition, validation, visualisation et approbation de la version à publier

O. V. - Conceptualisation, analyse initiale des données et première ébauche

M. E - Conceptualisation, analyse initiale des données et première ébauche

L. J. - Conceptualisation, analyse initiale des données et première ébauche

W. S. - Conceptualisation, révision de la première ébauche A. W. - Examen des données, recherche, révision de la version définitive

C. A. - Révision, rédaction, édition, approbation finale

\section{Conflit d'intérêts}

Aucun. 


\section{Remerciements}

L'Agence de la santé publique du Canada tient à remercier tous les fournisseurs de données pour leurs généreuses contributions et leur participation. La source des renseignements provient des programmes provinciaux et territoriaux portant sur le $\mathrm{VIH}$ et SIDA.

\section{Financement}

Ce travail a été réalisé grâce au soutien de l'Agence de la santé publique du Canada.

\section{Références}

1. Joint United Nations Programme on HIV/AIDS. UNAIDS Data 2018. UNAIDS; 2018. https://www.unaids.org/en/resources/ documents/2018/unaids-data-2018

2. Joint United Nations Programme on HIV/AIDS. HIV and aging - A special supplement to the UNAIDS report on the global AIDS epidemic UNAIDS; 2013. https://reliefweb.int/ report/world/hiv-and-aging-special-supplement-unaidsreport-global-aids-epidemic-2013

3. Joint United Nations Programme on HIV/AIDS. The GAP REPORT 2014: People aged 50 years and older. UNAIDS; 2014. https://www.unaids.org/sites/default/files/media_ asset/12_Peopleaged50yearsandolder.pdf

4. Wing EJ. HIV and aging. Int J Infect Dis 2016 Dec;53:61-8. DOI PubMed

5. Joint United Nations Programmed on HIV/AIDS. Get on the Fast-Track: The life cycle approach to HIV. UNAIDS: 2016. https://www.unaids.org/sites/default/files/media_asset/ Get-on-the-Fast-Track_en.pdf

6. Statistique Canada. Âge (en années) et âge moyen (127) et sexe (3) pour la population du Canada, provinces et territoires, régions métropolitaines de recensement et agglomérations de recensement, recensements de 2016 et 2011 - Données intégrales (100\%). Ottawa (ON): StatsCan; 2017. https://www12.statcan.gc.ca/ census-recensement/2016/dp-pd/dt-td/Rp-fra.cfm?TABID=2 \&LANG $=F \& A=R \& A P A T H=3 \& D E T A I L=0 \& D I M=0 \& F L=A \& F R E$ $\mathrm{E}=0 \& \mathrm{GC}=01 \& \mathrm{GL}=-1 \& \mathrm{GID}=1235625 \& \mathrm{GK}=1 \& \mathrm{GRP}=1 \& \mathrm{O}=\mathrm{D} \&$ $\mathrm{PID}=109523 \& \mathrm{PRID}=10 \& \mathrm{PTYPE}=109445 \& S=0 \& S H O W A L L=0$ $\& S U B=0 \&$ Temporal $=2016 \&$ THEME $=115 \& \mathrm{VID}=0 \& \mathrm{VNAMEE}=$ $\& V N A M E F=\& D 1=0 \& D 2=0 \& D 3=0 \& D 4=0 \& D 5=0 \& D 6=0$

7. Cahill S, Valadéz R. Growing older with HIV/AIDS: new public health challenges. Am J Public Health 2013 Mar;103(3):e7-15. DOl PubMed
8. Rodriguez-Penney AT, ludicello JE, Riggs PK, Doyle K, Ellis RJ, Letendre SL, Grant I, Woods SP; HIV Neurobehavioral Research Program HNRP Group. Co-morbidities in persons infected with HIV: increased burden with older age and negative effects on health-related quality of life. AIDS Patient Care STDS 2013 Jan;27(1):5-16. DOI PubMed

9. Önen NF, Overton ET, Seyfried W, Stumm ER, Snell M, Mondy K, Tebas P. Aging and HIV infection: a comparison between older HIV-infected persons and the general population. HIV Clin Trials 2010 Mar-Apr;11(2):100-9. DOl PubMed

10. Guaraldi G, Zona S, Brothers TD, Carli F, Stentarelli C, Dolci G, Santoro A, Beghetto B, Menozzi M, Mussini C, Falutz J. Aging with HIV vs. HIV seroconversion at older age: a diverse population with distinct comorbidity profiles. PLoS One 2015 Apr;10(4):e0118531. DOl PubMed

11. Agence santé publique du Canada. Le VIH et le sida au Canada - Rapport de surveillance en date du 31 décembre 2014. Ottawa (ON) : ASPC; 2015. https://www.canada.ca/fr/ sante-publique/services/publications/maladies-et-affections/ vih-et-sida-canada-rapport-surveillance-31-decembre-2014. html

12. Haddad N, Li JS, Totten S, McGuire M. Le VIH au Canada, Rapport de surveillance, 2017. Relevé des maladies transmissibles au Canada 2018;44(12):367-76. DOI

13. Statistique Canada. Estimations démographiques annuelles : Canada, provinces et territoires. 2017. Ottawa (ON): StatsCan ; 2018. https://www150.statcan.gc.ca/n1/fr/ catalogue/91-215-X

14. Youssef E, Wright J, Delpech V, Davies K, Brown A, Cooper $\mathrm{V}$, Sachikonye $\mathrm{M}$, de Visser R. Factors associated with testing for HIV in people aged $\geq 50$ years: a qualitative study. BMC Public Health 2018 Oct;18(1):1204. DOI PubMed

15. Tavoschi L, Gomes Dias J, Pharris A; EU/EEA HIV Surveillance Network. New HIV diagnoses among adults aged 50 years or older in 31 European countries, 2004-15: an analysis of surveillance data. Lancet HIV 2017 Nov;4(11):e514-21. DOl PubMed

16. Centers for Disease Control and Prevention. HIV Among People Aged 50 and Older. Atlanta (GA): CDC; 2016. https://www.cdc.gov/hiv/group/age/olderamericans/index. html

17. New York City Department of Health and Mental Hygiene. HIV Surveillance Annual Report, 2017. Queens (NY): NYC Health; 2018. https://www1.nyc.gov/assets/doh/downloads/ pdf/dires/hiv-surveillance-annualreport-2017.pdf

18. British Columbia Centre for Excellence in HIV/AIDS. HIV Monitoring Quarterly Report for British Columbia - Fourth Quarter 2018. Vancouver (BC): BC-CfE; 2019. http://www.stophivaids.ca/qmr/2018-Q4/\#/bc 
19. Ontario HIV Epidemiology and Surveillance Initiative. HIV testing in Ontario, 2016. Toronto (ON): OHESI; 2018. http://ohesi.ca/documents/OHESI-HIV-testing-i n-Ontario-in-2016.pdf

20. Flowers P, Estcourt C, Sonnenberg P, Burns F. HIV testing intervention development among men who have sex with men in the developed world. Sex Health 2017 Feb;14(1):80-8. DOI PubMed

21. Schick V, Herbenick D, Reece M, Sanders SA, Dodge B, Middlestadt SE, Fortenberry JD. Sexual behaviors, condom use, and sexual health of Americans over 50: implications for sexual health promotion for older adults. J Sex Med 2010 Oct;7 Suppl 5:315-29. DOI PubMed

22. Agence de la santé publique du Canada. Questions et réponses: Prévention des infections transmissibles sexuellement et par le sang chez les adultes âgés. Ottawa (ON) : ASPC. https://www.canada.ca/fr/ sante-publique/services/maladies-infectieuses/ sante-sexuelle-infections-transmissibles-sexuellement/ rapports-publications/questions-reponses-adultes.html

23. Youssef E, Cooper V, Delpech V, Davies K, Wright J. Barriers and facilitators to HIV testing in people age 50 and above: a systematic review. Clin Med (Lond) 2017 Dec;17(6):508-20. DOI PubMed
24. Harris TG, Rabkin M, El-Sadr WM. Achieving the fourth 90: healthy aging for people living with HIV. AIDS 2018 Jul;32(12):1563-9. DOl PubMed

25. Pilowsky DJ, Wu LT. Sexual risk behaviors and HIV risk among Americans aged 50 years or older: a review. Subst Abuse Rehabil 2015 Apr;6:51-60. DOI PubMed

26. Orel NA, Spence M, Steele J. Getting the message out to older adults: effective HIV health education risk reduction publications. J Appl Gerontol 2005;24(5):490-508. DOI

27. Drew O, Sherrard J. Sexually transmitted infections in the older woman. Menopause Int 2008 Sep;14(3):134-5. DOI PubMed

28. Negin J, Rozea A, Martiniuk AL. HIV behavioural interventions targeted towards older adults: a systematic review. BMC Public Health 2014 May;14:507. DOI PubMed

29. Agence de la santé publique du Canada. Summary: Estimates of HIV incidence, prevalence and Canada's progress on meeting the 90-90-90 HIV targets, 2016. Ottawa (ON) : ASPC. https://www.canada.ca/en/public-health/ services/publications/diseases-conditions/summary-estimate s-hiv-incidence-prevalence-canadas-progress-90-90-90.html

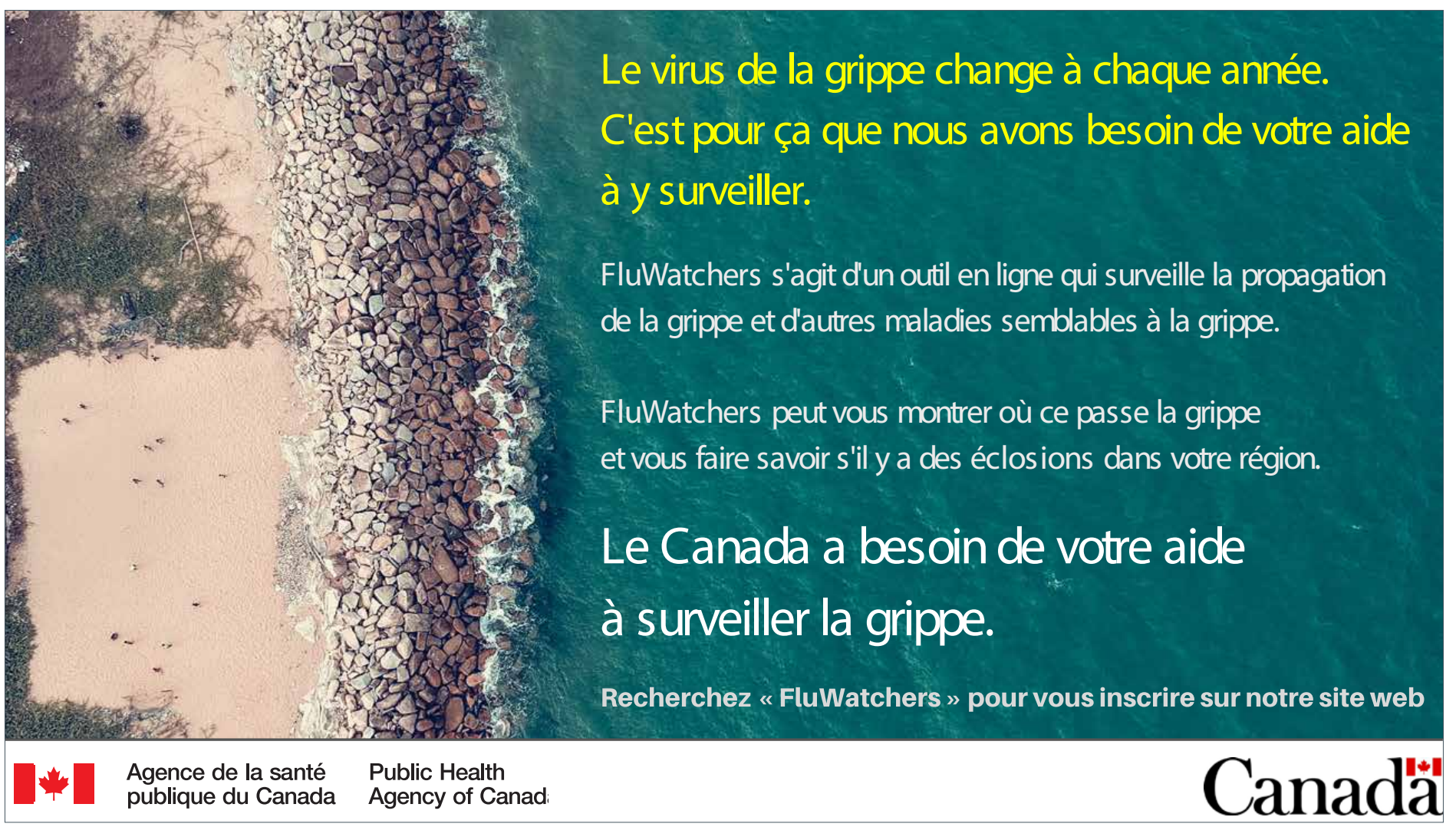

